\title{
Decreased LIPF expression is correlated with DGKA and predicts poor outcome of gastric cancer
}

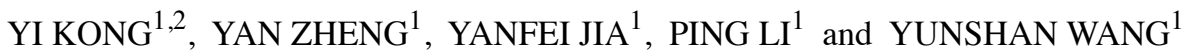 \\ ${ }^{1}$ Central Laboratory, Jinan Central Hospital Affiliated to Shandong University, Jinan, Shandong 250013; \\ ${ }^{2}$ Department of Clinical Laboratory, Jining First People's Hospital, Jining, Shandong 272011, P.R. China
}

Received February 21, 2016; Accepted April 18, 2016

DOI: 10.3892/or.2016.4989

\begin{abstract}
Gastric cancer (GC) is a common and deadly digestive tract tumor worldwide. Unfortunately, diagnosis of GC is usually confused and misleading because of atypical symptoms or incomplete complaints. Accordingly, exploring gene expression profile and identifying genes with analogical variance trend will bring new perspective into the diagnosis and treatment of GC. Herein, a RNA-Seq dataset from Caucasian GC and their matched non-cancerous samples [Gene Expression Omnibus (GEO): SRP049809] and datasets from four microarrays constituted with tumor and non-tumor tissues (GEO: GSE13911, GSE19826, GSE29272, GSE33335) were analyzed to explore the differentially expressed genes (DGEs). As a result, we identified a core set of 373 DGEs. Among these genes, we found that most downregulated genes were related to lipid-metabolic functions. Especially, the gastric lipase (LIPF) gene, which was connected with various lipid metabolism processes, was significantly decreased among all datasets. We then performed immunohistochemistry experiments using gastric tissue arrays to investigate the clinical effects, and the expression of a LIPF target gene, diacylglycerol kinase $\alpha$ (DGKA). Among the 90 samples of gastric adenocarcinoma, the LIPF and DGKA levels were both decreased in cancer tissues [LIPF, 59.1\% (53/90); DGKA, 77.8\% (70/90)] compared to normal tissues [LIPF, 94.4\% (85/90); DGKA, 90\% (81/90)]. The expression level of these two proteins in GC was associated with local invasion and disease stage. Cox regression identified high DGKA expression (HR, 0.49; 95\% CI, 0.26-0.94; $\mathrm{P}=0.03$ ) as a predictor of good prognosis and LNM status (HR, 4.63; 95\% CI, 1.39-15.51; $\mathrm{P}=0.01$ ) as a predictor of poor prognosis. Thus we speculated that LIPF-DGKA might serve as a potential possible biomarkers for diagnosis of $\mathrm{GC}$,
\end{abstract}

Correspondence to: Professor Yunshan Wang, Central Laboratory, Jinan Central Hospital Affiliated to Shandong University, 105 Jiefang Road, Jinan, Shandong 250013, P.R. China

E-mail: sdjnwys@sohu.com

Key words: RNA-Seq, microarray, gastric lipase, diacylglycerol kinase $\alpha$, gastric cancer, lipid metabolic aberration and their downregulation may bring new perspective into the investigation of GC prognosis.

\section{Introduction}

Gastric cancer (GC) is a highly lethal disease among cancers worldwide. Diagnosis of GC, however, is usually confusing and misleading due to atypical symptoms or unintelligible complaints. As we all know, various metabolic processes proceed in the stomach. Liver biomarkers in serum therefore have been introduced into diagnosis of GC recent years. For example, low serum pepsinogen levels imply the presence of atrophic gastritis, a premonition of intestinal type GC. Nevertheless the threshold values of serum pepsinogen levels were difficult to be determined, due to various factors (1). Thus, it is important to seek more metabolism-related genes to investigate $\mathrm{GC}$ through metabolic perspective. To assess crucial genes relevant to GC metabolism, we analyzed publicly available RNA-Seq datasets, composed of the comprehensive transcriptome profiles of 22 GC tissues and their non-cancerous counterpart samples, and performed an integrative analysis across different GC gene expression datasets. First, we identified a series of differentially expressed genes (DGEs) in tumor tissues. Then, we verified these genes by analyzing four independent microarray datasets from distinct microarray platforms using GC samples from different regions and ethnicities. Third, we performed gene function enrichment analysis on DEGs. As a result, we found that gastric lipase (LIPF), an enzyme which correlated with the digestion of dietary triglycerides in the gastrointestinal tract, was consistently downregulated in each dataset. Lipid metabolism is an established hallmark of cancer. Ether lipid levels have been shown to be elevated in tumors (2). Thus, our research may provide a novel significant perspective to investigate the relationship between lipid metabolic disturbance and GC progenesis.

\section{Materials and methods}

RNA-Seq datasets of GC patients. With the aim of obtaining an integrative series of DEGs in the stomach of GC patients compared with normal control tissues, we evaluated SRA-formatted files of RNA-Seq data from the Gene Expression Omnibus (GEO) database (3) under the accession 
number of SRP049809. It consisted of 22 separate samples derived from GC tissues and their matched non-cancerous samples, studied by the researchers in the National Cancer Center of Korea (NCCK) and the University of Texas MD Anderson Cancer Center (TMDACC). The dataset contained transcriptome of the gastric tissues in different locations isolated from eleven male and eleven female GC patients. In these experiments, total RNA was purified for library preparation (paired-end, 50 and $35 \mathrm{nt}$ ), template bead preparation and SOLiD v4.0 sequencing, following standard protocols provided by Life Technologies (Carlsbad, CA, USA).

After removing poly-A tails and low quality reads from the original data, we mapped short read data on the human genome reference sequence hg38 by using TopHat v2.0.14 (4). The number of mapped reads was calculated with HTSeq (5). Expression levels were evaluated using edgeR package (6). Significance of DEGs was expressed as Q-value, representing FDR-adjusted $\mathrm{P}<0.05$. Criteria of determining DGEs was set to two times fold changes.

Microarray datasets of GC patients. We investigated four distinct microarray datasets of GC retrieved from GEO dataset under accession nos. GSE13911, GSE19826, GSE29272, and GSE33335.

The GSE13911 dataset contained transcriptome of resected stomach tissues studied on an Affymetrix Human Genome U133 Plus 2.0 Array containing 47,000 transcripts (Affymetrix, Inc.), and the data were normalized by the Robust Multichip Average algorithm. The samples were collected by the researchers in IRBM (Merck Research Laboratories, Italy). There were $38 \mathrm{GCs}$ and normal control samples with or without microsatellite instability (MSI) which presented mismatch repair (MMR) inactivation or activation (age, 73.94 \pm 7.21; males, 15; females, 23). This dataset contained 6 patients with diffuse GC (age, 72.83 \pm 7.14 ; males, 1; females, 5), 26 patients with intestinal GC (age, 74.5 \pm 7.44 ; males, 12; females, 14), 4 patients with mixed GC (age, 68.6 \pm 6.4 ; males, 3 ; females, 1) and 2 patients with uncharacterized GC.

The GSE19826 dataset contained 27 transcriptome of GC samples studied on an Affymetrix Human Genome U133 Plus 2.0 Array with the same normalized method as GSE13911 above. The samples were collected by the researchers in the First People's Hospital affiliated to Shanghai Jiao Tong University, China. This dataset contained $24 \mathrm{GCs}$ and 3 normal control samples from patients with GC. There were three replicates in each histological stage. Other details, such as age and gender, were unavailable.

The GSE29272 dataset contained 268 samples of paired adjacent non-tumor tissues and cardia/non-cardia GC tissues. There were 62 patients with cardia GC and 72 patients with non-cardia GC. Samples were analyzed using the Affymetrix U133A Array. The cardia gastric dataset consisted of 1 male patient in stage I (age, 48), 56 patients in stage III (age, $58.73 \pm 10.29$; males, 39; females, 17), 5 patients in stage IV (age, 59.8 \pm 4.97 ; males, 5; females, 0 ). The non-cardia gastric dataset contained 4 patients in stage I (age, 55 \pm 10.10 ; males, 3; females, 1), 4 patients in stage II (age, 62 \pm 5.29 ; males, 4; females, 0), 59 patients in stage III (age, 54.71 \pm 10.07 ; males, 48; females, 11), 4 patients in stage IV (age, 51 \pm 16.02 ; males, 2 ; females, 2).
The GSE33335 dataset contained 50 samples of paired adjacent non-cancerous tissues and GC tissues. There were pairs of adjacent tissues and tumor tissues from 25 patients. These samples were analyzed with Affymetrix Human Exon 1.0 ST Array which contained 0.3 million probes. The dataset consisted of 7 patients in stage I (age, 67.43 \pm 11.15 ; males, 4; females, 3), 6 patients in stage II (age, 59.50 \pm 13.50 ; males, 4; females, 2), 6 patients in stage IV (age, 71.33 \pm 9.11 ; males, 4; females, 2) and a patient unclassified.

To assess the statistically significant DGEs between tumor and normal or adjacent non-tumor groups, we performed a paired t-test in LIMMA package (7-9).

Gene function enrichment analysis. Gene IDs of DEGs were submitted into the Functional Annotation Tool of Database for Annotation, Visualization and Integrated Discovery (DAVID) v6.7. David educes enriched gene ontology (GO) terms in the series of DEGs. The Fisher's exact test was employed to evaluate statistical significance. The significant threshold was set to $\mathrm{P}<0.05$ after Benjamini's correction. Then we searched the potential pathway related to GO terms enrichment to find the possible downstream or upstream genes.

Immunohistochemistry experiments. GC tissue sections containing HStm-Ade180Sur-02 (90 cancer cases) were provided by Outdo Biotech (Shanghai, China). Experiments were authorized by the Ethics Committee of Jinan central Hospital affiliated to Shandong University conforming with the Helsinki Declaration. Histological parameters were ascertained according to the criteria of the World Health Organization. Tumor lymph node metastasis classification by the Current International Union Against Cancer was employed to determine the pathologic stages.

Ninety gastric tumor tissue samples and their counterpart normal tissue samples were deparaffinized in xylene, sequentially antigen were retrieved with citrate buffer solution (Wuhan Boster Biological Technology, Ltd., Wuhan, Hubei, China). Endogenous peroxidase activity was inactivated by $0.3 \% \mathrm{H}_{2} \mathrm{O}_{2}$ for $15 \mathrm{~min}$ at room temperature. The sections were blocked with low lental serum for $30 \mathrm{~min}$ and subsequently incubated with primary antibodies [LIPF: Abcam, Cambridge, MA, USA; diacylglycerol kinase $\alpha$ (DGKA): Proteintech Group, Inc., Rosemont, IL, USA]. Incubation of secondary antibodies was performed with kits (ZSGB-BIO, Beijing, China). Antibody staining was visualized with DAB (D-5637; Sigma) and hematoxylin counterstain. Sections with 5\% labeled cells were scored as 0 ; with 5-25\% labeled cells as 1; with $26-50 \%$ labeled cells as 2 ; with $50-80 \%$ labeled cells as 3; and with $>80 \%$ labeled cells as 4 . The staining intensity was scored similarly, with 0 indicating negative staining, 0.5 weakly positive, 1 moderately positive and 2 strongly positive. The scores for the percentage of positive tumor cells and staining intensity were multiplied to generate an immunoreactive score for each specimen. Samples with scores $\geq 3$ were considered as high expression while those with scores $<3$ were considered as low expression.

Statistical analysis. The correlation of LIPF and its potential relevant genes, DGKA clinicopathological char- 
A

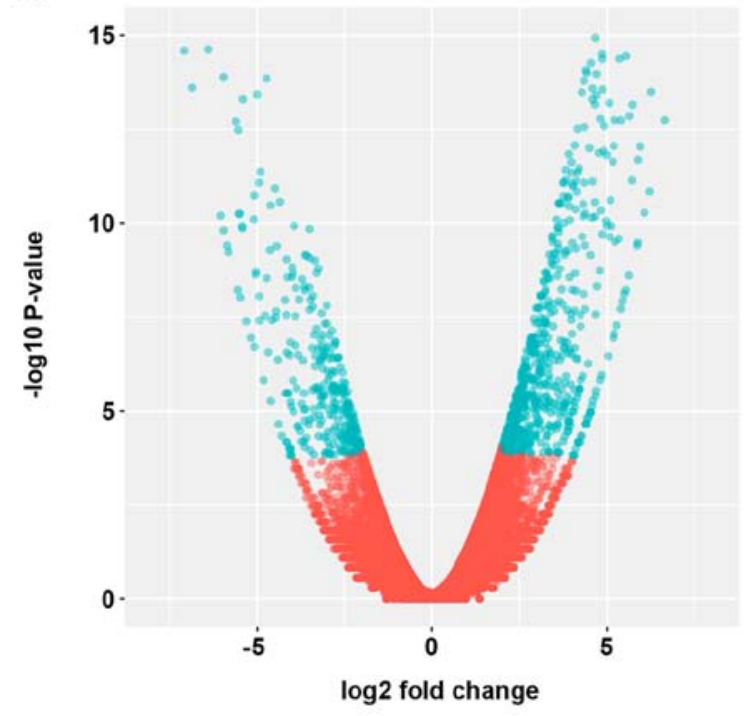

C

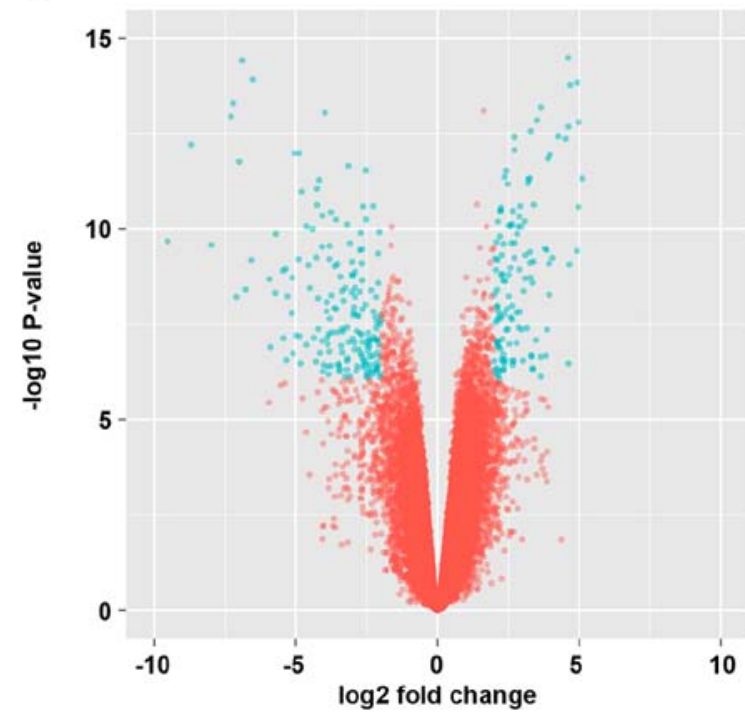

B

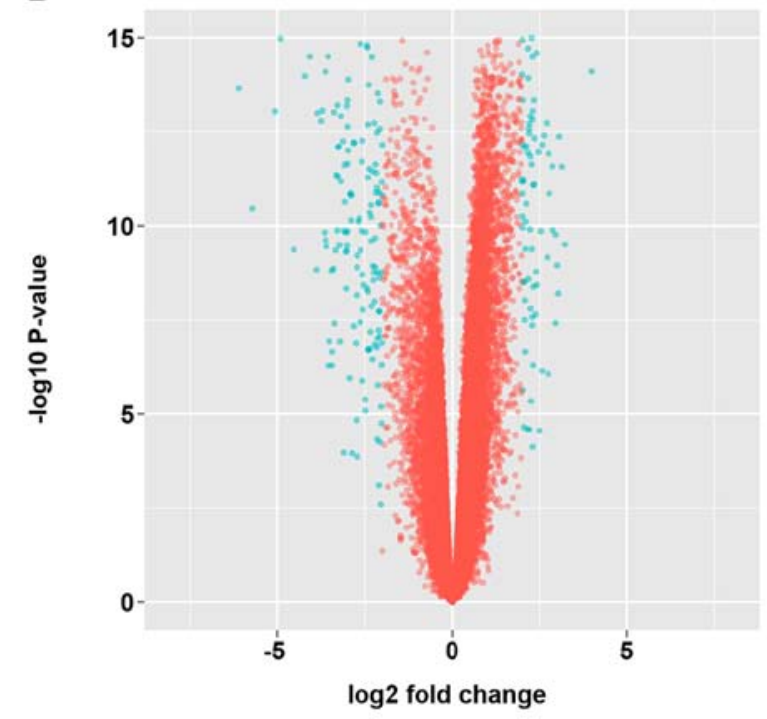

D

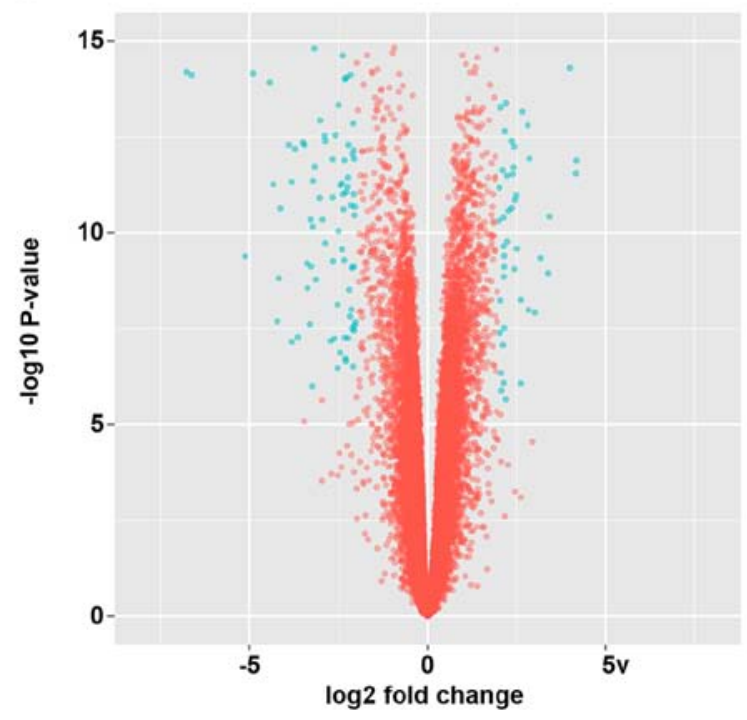

Figure 1. The panorama of gene expression levels in distinct datasets. (A) SRP049809; (B) GSE13911; (C) GSE29272; (D) GSE33335. Genes with significantly differentiated expression are marked with blue color. The Q-value in RNA-Seq dataset and adjusted P-value in microarray were employed to evaluate the significant level ( $\mathrm{P}$ or $\mathrm{Q}<0.05)$.

acteristics were evaluated by the Spearman's rank test and $\chi^{2}$ test. Expression level of these biomarkers was investigated through Mann-Whitney test. Kaplan-Meier survival curves were calculated using the log-rank test. Multivariate Cox regression analysis was employed to investigate the potential prognostic factors for survival in patients with GC. $\mathrm{P}<0.05$ was considered statistically significant. The statistical calculation was performed in $\mathrm{R}$ language package.

\section{Results}

RNA-Seq and microarray data analysis of GC tissues. Based on RNA-Seq data analyzed with TopHat and HTSeq, we studied transcriptome of the stomach samples from resected tumor and normal tissues collected by NCCA and TMDACC. Among them, we identified a series of DEGs that satisfied $\mathrm{Q}$-value (FDR-corrected P-value) $<0.05$ and $\mid \log \mathrm{FCl}>2$ times, when compared between GC and normal groups. Sequentially, we divided these DEG into 476 upregulated genes and 222 downregulated genes in GC (Fig. 1A). Tumor progression always correlated with overexpression of oncogenes and inactivation of anti-oncogenes. Downregulating of anti-oncogenes may play a crucial role in tumorigenesis (10).

Previous research was mainly focused on upregulated genes, lacking of deep data evaluations on download genes. DEGs were assessed and refined to obtain a core set which reduced redundancies, according to Q-values, fold change values and functions. Obviously, the expression of LIPF, an enzyme involved in the digestion of dietary triglycerides in the gastrointestinal tract, and responsible for $30 \%$ of fat digestion processes occurring in human, was greatly reduced at fold changes of $-2.55(\mathrm{Q}=1.62 \mathrm{E}-04)$.

To confirm the results of RNA-Seq data analysis, we studied four microarray datasets of GC tissues numbered GSE13911, 
Table I. Top rank differentially expressed genes among distinct datasets.

\begin{tabular}{|c|c|c|c|}
\hline Gene ID & Gene name & Full name & Average $\log \mathrm{FC}$ \\
\hline 2694 & GIF & Gastric intrinsic factor & -6.586 \\
\hline 496 & ATP4B & ATPase, $\mathrm{H}^{+} / \mathrm{K}^{+}$exchanging, $\beta$ polypeptide & -6.54044 \\
\hline 8513 & $L I P F$ & Gastric lipase & -6.438 \\
\hline 56287 & GKN1 & Gastrokine 1 & -5.722 \\
\hline 495 & ATP4A & ATPase, $\mathrm{H}^{+} / \mathrm{K}^{+}$exchanging, $\alpha$ polypeptide & -5.114 \\
\hline 2104 & ESRRG & Estrogen-related receptor $\gamma$ & -4.65 \\
\hline 643834 & PGA3 & Pepsinogen 3, group I (pepsinogen A) & -4.426 \\
\hline 10690 & FUT9 & Fucosyltransferase 9 ( $\alpha 1,3$-fucosyltransferase) & -4.406 \\
\hline 9992 & KCNE2 & Potassium voltage-gated channel, Isk-related family, member 2 & -4.372 \\
\hline 5225 & PGC & Progastricsin (pepsinogen C) & -4.362 \\
\hline 27159 & CHIA & Chitinase, acidic & -4.322 \\
\hline 200504 & GKN2 & Gastrokine 2 & -4.318 \\
\hline 57016 & AKR1B10 & Aldo-keto reductase family 1, member B10 (aldose reductase) & -3.92 \\
\hline 1358 & CPA2 & Carboxypeptidase A2 (pancreatic) & -3.818 \\
\hline 3624 & INHBA & Inhibin $\beta A$ & 4.235 \\
\hline 1469 & CST1 & Cystatin SN & 3.69 \\
\hline 4102 & MAGEA3 & Melanoma antigen family A, 3 & 3.5775 \\
\hline 6696 & SPP1 & Secreted phosphoprotein 1 & 3.412 \\
\hline 23213 & SULF1 & Sulfatase 1 & 3.078 \\
\hline
\end{tabular}

GSE19826, GSE29272 and GSE33335. First, GSE13911, composed of transcriptome of GC tissues with and without MSI isolated in European patients, were investigated. We identified a series of 260 DEGs, including 166 downregulated and 94 upregulated genes. Because previous research was mainly focused on upregulated, instead of downregulated, genes we intended to obtain a deep panorama of downregulated gene networks. Conspicuously, expression of LIPF decreased at fold change equal to -6.09 times in gastric tumor tissues $(\mathrm{P}=6.05 \mathrm{E}-12)$. In contrast, other lipase did not show significant expression difference ( $\mathrm{P}>0.05)$ between $\mathrm{GC}$ and $\mathrm{NC}$ in this dataset.

Sequentially, we analyzed datasets of transcriptome isolated from adjacent normal/tumor-matched gastric tissues in Asian patients (GSE19826 on Human Genome U133 Plus 2.0 Array; GSE29272 on Human Genome U133A Array and GSE33335 on Human Exon 1.0 ST Array). From the GSE19826 dataset, 378 DEGs were downregulated in the gastric tumor tissues with the $\log \mathrm{FCl}>2$ times of GC versus NC. The expression of LIPF decreased at fold change of -10.47 times $(\mathrm{P}=1.15 \mathrm{E}-13)$. From the GSE29272 dataset 75 downregulated DEGs were assessed in the gastric cardia and non-cardiac adenocarcinomas from Chinese patients. Analogically, the expression of LIPF decreased in GC at fold change of -5.23 times $(\mathrm{P}=4.01 \mathrm{E}-52)$. From the GSE33335 dataset, we identified 161 DEGs which constituted 107 downregulated and 54 upregulated genes. The expression of LIPF decreased in GC at fold change equal to -7.86 times $(\mathrm{P}=3.22 \mathrm{E}-17)$. The outline of the spread of DEGs in each dataset is shown in Fig. 1. We combined RNA-Seq and microarray data to select a core set of 373 DEGs. Taken together, from these transcriptome data, the expression of LIPF was significantly decreased in the GC tissues in spite of different location in the stomach, microarray platforms, or ethnicity of the samples.
Top rank DEGs which possessed statistical significance are listed in Table I. The average $\log F C$ values were calculated to obtain a comprehensive assessment on distinct datasets.

Gene function annotation. DAVID annotation demonstrated that most downregulated genes in gastric tumor tissues were enriched in GO terms of 'lipid homeostasis' (GO: 0055088, $\mathrm{P}=3.3 \mathrm{E}-2$ ), 'organic ether metabolic process' (GO: 0018904, $\mathrm{P}=1.8 \mathrm{E}-3$ ), 'lipid catabolic process' (GO: 0016042, $\mathrm{P}=5.6 \mathrm{E}-5)$.

LIPF was related with the signal pathways 'glycerolipid metabolism' (hsa00561), 'metabolic pathways' (hsa01100) and 'fat digestion and absorption' (hsa04975) in KEGG database. DGKA, a potential downstream gene of LIPF was reported to play an important role in tumorigenesis (11). To explore the protein expression levels of LIPF and DGKA in GC, we performed immunohistochemistry in GC and adjacent non-tumor tissues and analyzed the correlation between LIPF, DGKA expression and clinicophathological characteristics of GC patients.

Results showed that LIPF protein was mainly located in cell membranes and cytoplasm in gastric cells (Fig. 2). Among $90 \mathrm{GC}$ and adjacent normal tissues, LIPF was frequently expressed in normal tissues $(94.4 \%, 85 / 90)$, while in GC tissues the positive percent is $59.1 \%$ (53/90). A significant difference was observed between these two groups $(\mathrm{P}<0.001)$. Additionally, higher level of LIPF expression was detected in early stage of GC (stage I, II; mean, 2.000 $\pm 0.1905, \mathrm{~N}=32$ ), while in advanced stage of GC the expression was lower (stage III, IV; mean, 0.5517 $\pm 0.1098, \mathrm{~N}=58$ ) (Fig. 3A, Table II). Analogical analysis was also performed on IHC results from DGKA, and the results are listed in Table II. The positive rate of DGKA decreased to $77.8 \%$ (70/90) in GC from 90\% (81/90) in normal tissues. There was a significant difference between 
Table II. Correlation between expression of LIPF, DGKA and clinicopathological features of GC patients.

\begin{tabular}{|c|c|c|c|c|c|c|c|c|}
\hline Characteristics & $\begin{array}{l}\text { LIPF high } \\
\text { expression }\end{array}$ & $\begin{array}{l}\text { LIPF low } \\
\text { expression }\end{array}$ & $\begin{array}{c}\chi^{2} \text { or Fisher's } \\
\text { exact test }\end{array}$ & P-value & $\begin{array}{l}\text { DGKA high } \\
\text { expression }\end{array}$ & $\begin{array}{l}\text { DGKA low } \\
\text { expression }\end{array}$ & $\begin{array}{c}\chi^{2} \text { or Fisher's } \\
\text { exact test }\end{array}$ & P-value \\
\hline Age (years) & & & $2.18 \mathrm{E}-30$ & 1.0 & & & 0.014 & 0.91 \\
\hline$<60$ & 5 & 26 & & & 9 & 22 & & \\
\hline$\geq 60$ & 10 & 49 & & & 15 & 44 & & \\
\hline Gender & & & & 1.0 & & & $2.94 \mathrm{E}-4$ & 0.99 \\
\hline Male & 10 & 52 & & & 16 & 46 & & \\
\hline Female & 4 & 24 & & & 8 & 20 & & \\
\hline Local invasion & & & 10.03 & 0.002 & & & & 0.0069 \\
\hline $\mathrm{T} 1+\mathrm{T} 2$ & 6 & 5 & & & 7 & 4 & & \\
\hline $\mathrm{T} 3+\mathrm{T} 4$ & 9 & 70 & & & 17 & 62 & & \\
\hline Nodal status & & & 2.17 & 0.14 & & & 2.27 & 0.13 \\
\hline Positive & 8 & 57 & & & 14 & 51 & & \\
\hline Negative & 7 & 18 & & & 10 & 15 & & \\
\hline Stage of disease & & & 4.53 & 0.033 & & & 4.15 & 0.041 \\
\hline $\mathrm{I}+\mathrm{II}$ & 10 & 25 & & & 14 & 21 & & \\
\hline $\mathrm{III}+\mathrm{IV}$ & 5 & 50 & & & 10 & 45 & & \\
\hline Distant metastasis & & & & 1.0 & & & & 1.0 \\
\hline M0 & 15 & 74 & & & 24 & 65 & & \\
\hline M1 & 0 & 1 & & & 0 & 1 & & \\
\hline $\begin{array}{l}\text { Depth of tumor } \\
\text { invasion }\end{array}$ & & & 0.33 & 0.031 & & & 0.15 & 0.70 \\
\hline $\begin{array}{l}\text { Mucosa, submucosa, } \\
\text { muscularis propria, } \\
\text { subserosa }\end{array}$ & 14 & 47 & & & 15 & 46 & & \\
\hline $\begin{array}{l}\text { Penetration of serosa, } \\
\text { adjacent structures }\end{array}$ & 1 & 28 & & & 9 & 20 & & \\
\hline
\end{tabular}

LIPF, gastric lipase; DGKA, diacylglycerol kinase $\alpha$; GC, gastric cancer.
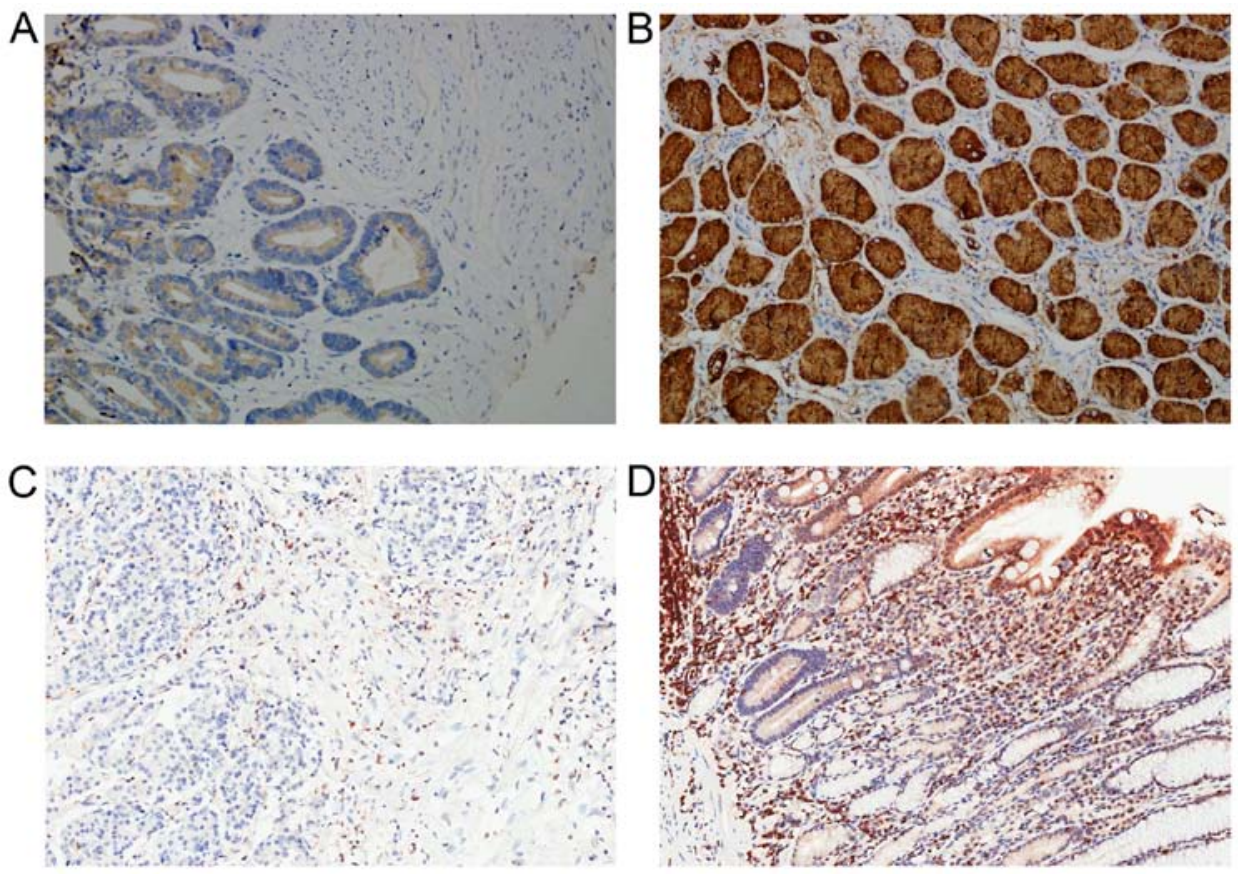

Figure 2. Immunohistochemical analyses of gastric lipase (LIPF) and diacylglycerol kinase $\alpha$ (DGKA) in gastric cancer (GC) and normal tissues. (A) LIPF expression in GC; (B) LIPF expression in normal tissues; (C) DGKA expression in GC; (D) DGKA expression in normal tissues. 
A
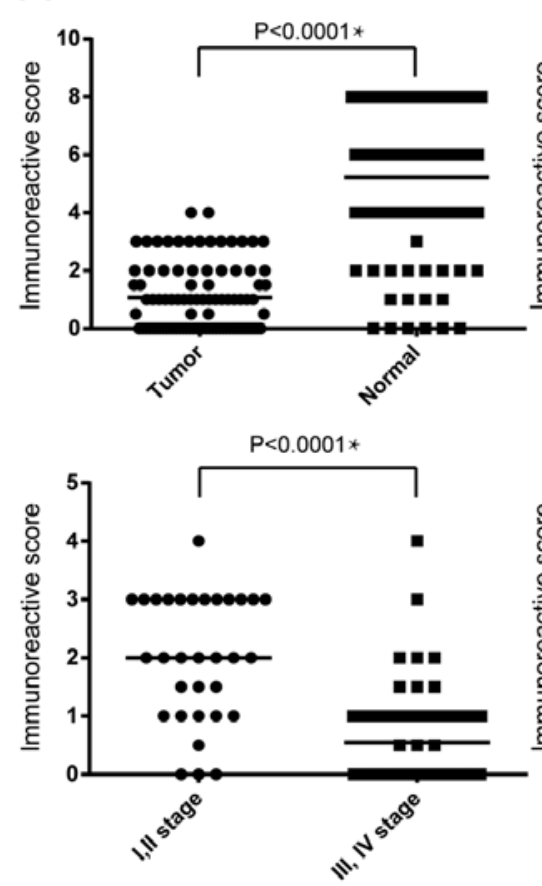

LIPF
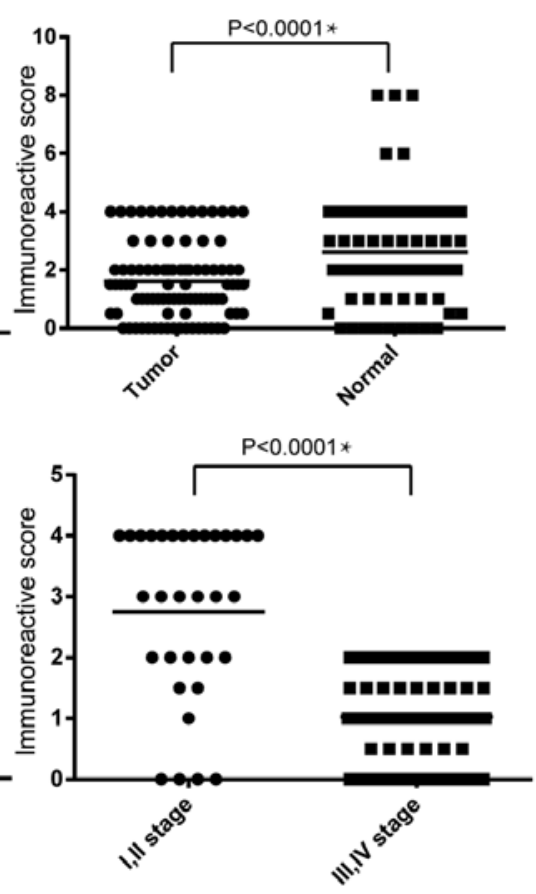

DGKA
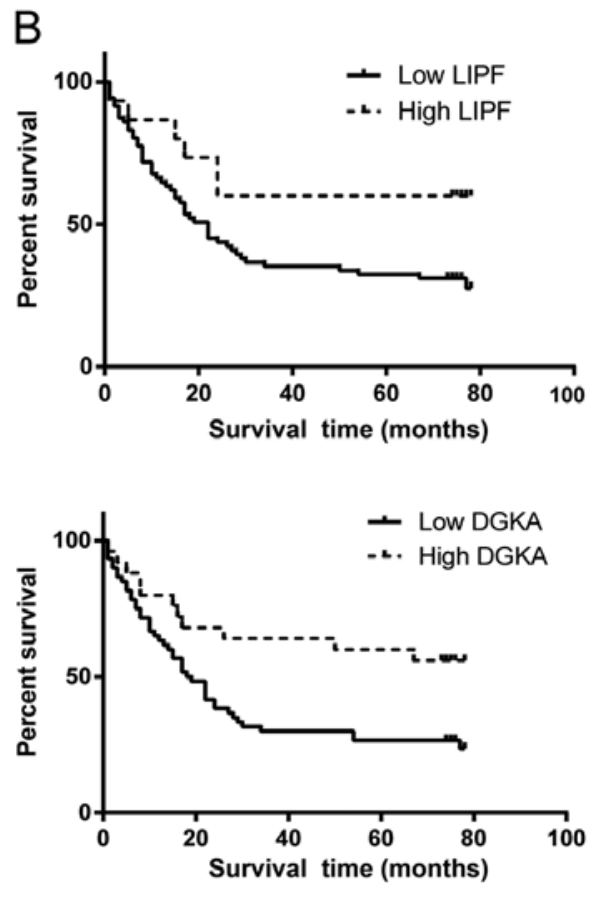

Figure 3. Expression level and survival analysis of gastric lipase (LIPF) and diacylglycerol kinase $\alpha$ (DGKA). (A) The comparison of immunoreactive score for LIPF and DGKA between gastric cancer (GC) and normal tissues, disease early stage (I, II) and late stage (III, IV). (B) Expression of LIPF and DGKA was assessed by immunohistochemistry and survival curves in patients with CG according to expression levels.

GC and normal tissues $(\mathrm{P}<0.05)$. Obvious disparity was found comparing early stage $(2.750 \pm 0.2490, \mathrm{~N}=32)$ and late stage $(1.078 \pm 0.1056, \mathrm{~N}=58) \mathrm{GC}$.

Survival analysis. The 90 patients were predominantly males $(68.9 \%)$ with a performance status of 0 (females) or 1 (males). The median age was 67 years (range, $42-83$ years). A total of $35.5 \%$ of patients belonged to early stage according to the AJCC classification strategy (stage I and II) while the other patients belonged to late stage (stage III and IV). Twelve percent of patients had low level local invasion (T1 and T2) while the other $88 \%$ of patients had high level local invasion (Table III). Median follow-up time was 22 months (range from 1 to 78 months). Among these patients, 56 (65.8\%) died during the follow-up period. In univariate analysis of prognostic value of clinical factors for progression-free survival, poor prognosis was associated with lymph node metastasis. Stage III and IV diseases were associated with a poor prognosis compared with stage I and II diseases $\left(\mathrm{P}=2.02 \times 10^{-5}\right)$. A better prognosis was associated with high DGKA expression type as compared with low DGKA expression type (Fig. 3B). A moderate increase in LIPF expression was associated with a decreased risk but without reaching statistical significance. Gender $(\mathrm{P}=0.69)$, age $(\mathrm{P}=0.40)$ and local invasion status $(\mathrm{P}=0.13)$ did not reach a significance for predicting prognostic value of clinical factors for progression-free survival. In order to select the most appropriate variables, a method following stepwise Akaike's Information Criterion (AIC) was employed. A group of variables with the lowest AIC value was chosen for subsequent multivariate Cox regres- sion analysis. In the multivariate Cox regression analysis, lymph node metastasis $\left(\mathrm{P}=1.97 \times 10^{-5}\right)$ was also associated with a poor prognosis. High expression of DGKA means a decrease of risk. Compared to univariate analysis, the patient age $(\mathrm{P}=0.028)$ was significantly associated with a low risk (Table IV).

\section{Discussion}

Recently, massive transcriptome studies of GC have been published on GEO database. Analysis of these data will provide important insights into the mechanisms underlying the progression of GC, and give more hints to find novel diagnostic and therapeutic methods for GC. We investigated the RNA-Seq datasets of GC transcriptome derived from NCCK and TMDACC, and identified a core set of 373 DEGs in GC vs. adjacent normal tissues. Then, we combined four independent microarray datasets to filtrate these DEGs into a core set. The downregulated genes in GC tissues, related with digestion, lipid catabolic process, lipid binding, lipase inhibitor activity, steroid binding by GO and pathway analysis. Previous research focused on upregulated gene in GC (12), however, downregulated genes in this dataset demonstrated more tissue-specific patterns in GO analysis. The downregulated genes in GC tissues were found to be related with digestion, lipid catabolic process, lipid binding, lipase inhibitor activity, steroid binding via GO and pathway analysis. We compared the results of RNA-Seq data with four different microarray datasets of GC tissues from different regions, microarray platforms and ethnicities. Based on 
Table III. Clinical characteristics of GC tissues.

\begin{tabular}{|c|c|c|}
\hline $\begin{array}{l}\text { Clinicopathological } \\
\text { features }\end{array}$ & No. & $\begin{array}{l}\text { Percentage } \\
\quad(\%)\end{array}$ \\
\hline \multicolumn{3}{|l|}{ Age (years) } \\
\hline$<60$ & 31 & 34.4 \\
\hline$\geq 60$ & 59 & 65.6 \\
\hline \multicolumn{3}{|l|}{ Gender } \\
\hline Male & 62 & 68.9 \\
\hline Female & 28 & 31.1 \\
\hline \multicolumn{3}{|l|}{ Tumor size (cm) } \\
\hline$<10$ & 79 & 87.8 \\
\hline$\geq 10$ & 11 & 12.2 \\
\hline \multicolumn{3}{|l|}{ Tumor site } \\
\hline Gastric cardia & 11 & 12.2 \\
\hline Body & 24 & 26.7 \\
\hline Gastric antrum & 38 & 42.2 \\
\hline Gastric notch & 9 & 10 \\
\hline Other & 8 & 8.8 \\
\hline \multicolumn{3}{|l|}{ Tumor stage } \\
\hline I & 4 & 4.4 \\
\hline II & 28 & 31.1 \\
\hline III & 52 & 57.4 \\
\hline IV & 1 & 1.1 \\
\hline Follow-up time (months) & $37(1-78)$ & \\
\hline \multicolumn{3}{|l|}{ Prognosis } \\
\hline Alive & 34 & 37.8 \\
\hline Dead & 56 & 62.2 \\
\hline Patients lived for 5 years & 31 & 34.4 \\
\hline \multicolumn{3}{|l|}{ Nodal status } \\
\hline Positive & 65 & 72.2 \\
\hline Negative & 25 & 27.8 \\
\hline \multicolumn{3}{|l|}{ Pathological type } \\
\hline Adenocarcinoma & 90 & 100 \\
\hline
\end{tabular}

a comprehensive assessment, we identified that LIPF was consistently downregulated among these datasets.

LIPF, an enzyme involved in the digestion of dietary triglycerides in the gastrointestinal tract, is responsible for $30 \%$ of fat digestion processes occurring in human. It hydrolyzes the ester bonds of triglycerides under acidic $\mathrm{pH}$ conditions. The conserved lipase family is composed of a series of paralogs with tissue-specificity, such as LIPA, LIPM. LIPF plays distinct roles in neutral lipid metabolism. It is usually secreted by gastric chief cells in the fundic mucosa of the stomach. The deficiency of LIPF and its paralog, LIPA, is associated with Wolman disease and cholesteryl ester storage disease (13). Recruitment of LIPF by free fatty acid-rich particles inhibited triacylglycerol hydrolysis (14). LIPF was involved in two important metabolic pathways, glycerolipid metabolism (KEGG: hsa00561) and fat digestion and absorption (KEGG: ko04975). The downstream gene of LIPF, DGKA, was reported to play a crucial role in secretion of Fas ligand-containing exosomes (15). Study showed that exosomes release was positively correlated with the invasiveness of ovarian cancer, which brought potential for diagnosis in peripheral blood detection (16). Different expression levels of LIPF and its target genes in GC may suggest a novel perspective upon relationship between tumor progression and lipid metabolic disorder. These genes may provide metabolic insights for GC investigation. Results of immunohistochemistry verified that the expression of LIPF was significantly decreased and the expression level was related to the pathological stage of GC. Compared with early stage, samples of stage III and IV had a lower expression of LIPF (Fig. 3A). We also demonstrated that the local invasion, stage of disease, and depth of tumor invasion correlated significantly with LIPF ( $\mathrm{P}<0.05$, Table II). Besides, lipid metabolism was involved in the initiation and/or progression of cancer-associated cachexia (17). In fatty acid metabolism of aggressive human cancer cells, oxidative pathways were reduced, instead, pathways for generating structural and signaling lipids were increased (18). Decreased LIPF level may play an important role in this lipogenic conversion.

Diacylglycerol kinases (DGKs) are composed by 10 enzymes, which metabolize 1,2-diacylglycerol (DAG) to produce phosphatidic acid (PA). DGKA is a key regulator of

Table IV. Multivariate Cox regression analysis of potential prognostic factors for survival in patients with GC.

\begin{tabular}{lccc}
\hline & \multicolumn{2}{c}{ Univariate analysis } & \multicolumn{1}{c}{ Multivariate analysis } \\
\cline { 2 - 3 } Variables & HR $(95 \% \mathrm{CI})$ & P-value & HR (95\% CI) \\
\hline Gender (male vs. female) & $0.51-1.55$ & 0.69 & 0.02 \\
Age ( $\geq 60$ vs. $<60$ years) & $0.44-1.36$ & 0.40 & $0.29-0.93$ \\
LNM status (yes vs. no) & $2.58-16.54$ & $7.19 \mathrm{E}-05$ & $1.39-15.51$ \\
DGKA (high vs. low) & $0.21-0.84$ & 0.01 & $0.26-0.94$ \\
LIPF (high vs. low) & $0.15-0.95$ & 0.07 & 0.03 \\
Tumor status (T3+T4 vs. T1+T2) & $0.76-7.79$ & 0.13 & \\
Stage (III+IV vs. I+II) & $2.19-8.31$ & $2.02 \mathrm{E}-05$ & \\
\hline
\end{tabular}

GC, gastric cancer; DGKA, diacylglycerol kinase $\alpha$; LIPF, gastric lipase. 


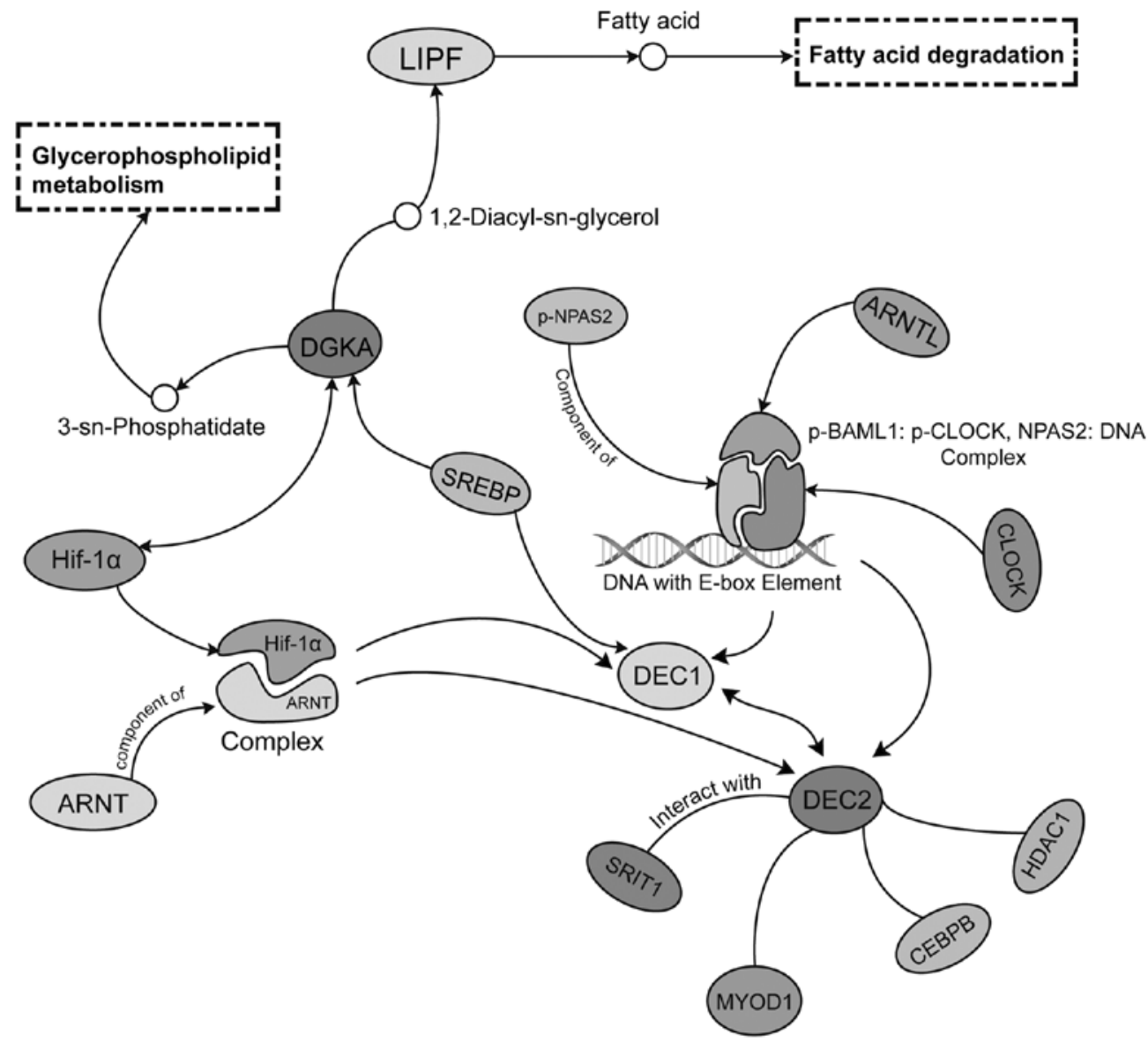

Figure 4. Potential regulating signal pathway related to gastric lipase (LIPF) and diacylglycerol kinase $\alpha$ (DGKA) in gastric cancer (GC). LIPF degrade 1,2-diacyl-sn-glycerol into fatty acid for subsequent fatty degradation. DGKA phosphorylated this substrate of LIPF for glycerophospholipid metabolism. DGKA regulated Hif- $1 \alpha$ and mTOR-SREBP pathways. Interestingly it also received feedback regulation from Hif-1 $\alpha$ and SREBP. Analogical regulation manner existed in DEC1/2, regulation targets of Hif-1 $\alpha$ and SREBP. They played a crucial role in circadian rhythm, tumor progenesis, which also regulated lipid metabolism. Theoretically, tumor cells demanded more lipids for membrane assemblage due to their exuberant proliferation.

the polarized secretion of exosomes, which had a particular lipid and protein content (19). Takeishi et al reported that DGKA enhanced hepatocellular carcinoma progression through activating Ras-Raf-MEK-ERK pathway (11), playing a crucial role in dysfunction of human tumor-infiltrating $\mathrm{CD}^{+} \mathrm{T}$ cells $(20)$. DGKA was also considered as a potential therapeutic target in glioblastoma cell lines, such as U87MG and U251 (21). In addition, downregulation of DGKA caused toxicity through key oncogenic pathways, including mTOR-SREBP and Hif-1 $\alpha$. Moreover, overexpression of mTOR and Hif- $1 \alpha$ rescued the toxicity from DGKA knockdown and inhibition.

We also compared the difference of DGKA expression between GC and normal tissues. Expression level of DGK correlated positively with LIPF in Spearman's rank test $(\mathrm{P}<0.05)$. Our results showed that the difference of DGKA expression correlated with local invasion, stage of disease $(\mathrm{P}<0.05)$.

Previous research indicated that high expression level of DGKA was related to poor prognosis, in other words, low expression was related to better prognosis (11). However, our results indicated an alternative regulating pattern in GC. The Cox regression results implied a protective effect of high DGKA rather than a poor risk (HR, 0.19-0.79;
95\% CI; $\mathrm{P}=0.041)$. Analogically, the reversed regulating pattern was also observed in DEC1, another important regulating target of Hif- $1 \alpha$ in GC (Fig. 4). Low DEC1 expression was associated with poor histological differentiation and malignancy progression in hepatocellular carcinoma, while high DEC1 expression was associated with poor histological differentiation in gastric carcinoma and non-small cell lung cancer (22-24). Interestingly, the regulating targets of DGKA, Hif- $1 \alpha$ and SERBP, also served as mediators of DEC1 and its paralog, the DEC2. This interdependency implied a potential regulating network, connecting glycerolipid metabolism with hypoxia-dependent circadian clock derangement. The DGKA expression demonstrated tissue-specificity, which indicated different regulation mechanisms in various organisms.

In conclusion, bioinformatics data mining on RNA-Seq and microarray datasets of GC indicated that LIPF was a downregulated DEG in GC. IHC results confirmed that the downregulation of LIPF and its target gene, DGKA, which might reduce the lipid decompose metabolism, provided lipid resource to the growth and division of cancer cells. Thus, we speculate that LIPF might play a crucial role in the development of GC. Further experiments need to be done to investigate the detail mechanisms. 


\section{Acknowledgements}

This study was financially supported by the National Natural Science Foundation of China (NSFC nos. 81000869 and 81272588) and Project 973 (grant nos. 2012CB966503 and 2012CB966504).

\section{References}

1. Ohata H, Oka M, Yanaoka K, Shimizu Y, Mukoubayashi C, Mugitani K, Iwane M, Nakamura $\mathrm{H}$, Tamai $\mathrm{H}$, Arii $\mathrm{K}$, et al: Gastric cancer screening of a high-risk population in Japan using serum pepsinogen and barium digital radiography. Cancer Sci 96: 713-720, 2005.

2. Benjamin DI, Cozzo A, Ji X, Roberts LS, Louie SM, Mulvihill MM, Luo K and Nomura DK: Ether lipid generating enzyme AGPS alters the balance of structural and signaling lipids to fuel cancer pathogenicity. Proc Natl Acad Sci USA 110: 14912-14917, 2013.

3. Barrett T, Wilhite SE, Ledoux P, Evangelista C, Kim IF, Tomashevsky M, Marshall KA, Phillippy KH, Sherman PM, Holko M, et al: NCBI GEO: Archive for functional genomics data sets - update. Nucleic Acids Res 41 (D1): D991-D995, 2013.

4. Trapnell C, Pachter L and Salzberg SL: TopHat: Discovering splice junctions with RNA-Seq. Bioinformatics 25: 1105-1111, 2009.

5. Anders S, Pyl PT and Huber W: HTSeq - a Python framework to work with high-throughput sequencing data. Bioinformatics 31 : 166-169, 2015.

6. Robinson MD and Smyth GK: Moderated statistical tests for assessing differences in tag abundance. Bioinformatics 23: 2881-2887, 2007.

7. Carvalho BS and Irizarry RA: A framework for oligonucleotide microarray preprocessing. Bioinformatics 26: 2363-2367, 2010.

8. Bolstad BM,Irizarry RA, Astrand M and Speed TP: A comparison of normalization methods for high density oligonucleotide array data based on variance and bias. Bioinformatics 19: 185-193, 2003.

9. Ritchie ME, Phipson B, Wu D, Hu Y, Law CW, Shi W and Smyth GK: limma powers differential expression analyses for RNA-sequencing and microarray studies. Nucleic Acids Res 43: e47-e47, 2015.

10. Johnson TM and Attardi LD: Dissecting p53 tumor suppressor function in vivo through the analysis of genetically modified mice. Cell Death Differ 13: 902-908, 2006.

11. Takeishi K, Taketomi A, Shirabe K, Toshima T, Motomura T, Ikegami T, Yoshizumi T, Sakane F and Maehara Y: Diacylglycerol kinase alpha enhances hepatocellular carcinoma progression by activation of Ras-Raf-MEK-ERK pathway. J Hepatol 57: 77-83, 2012.

12. Chang HR, Nam S, Kook MC, Kim KT, Liu X, Yao H, Jung HR, Lemos R Jr, Seo HH, Park HS, et al: HNF4a is a therapeutic target that links AMPK to WNT signalling in early-stage gastric cancer. Gut 65: 19-32, 2016.
13. Tylki-Szymańska A and Jurecka A: Lysosomal acid lipase deficiency: Wolman disease and cholesteryl ester storage disease. Pril (Makedon Akad Nauk Umet Odd Med Nauki) 35: 99-106, 2014.

14. Pafumi Y, Lairon D, de la Porte PL, Juhel C, Storch J, Hamosh M and Armand M: Mechanisms of inhibition of triacylglycerol hydrolysis by human gastric lipase. J Biol Chem 277: 28070-28079, 2002.

15. Alonso R, Mazzeo C, Rodriguez MC, Marsh M, Fraile-Ramos A, Calvo V, Avila-Flores A, Merida I and Izquierdo M: Diacylglycerol kinase $\alpha$ regulates the formation and polarisation of mature multivesicular bodies involved in the secretion of Fas ligand-containing exosomes in T lymphocytes. Cell Death Differ 18: 1161-1173, 2011.

16. Kobayashi M, Salomon C, Tapia J, Illanes SE, Mitchell MD and Rice GE: Ovarian cancer cell invasiveness is associated with discordant exosomal sequestration of Let-7 miRNA and miR-200. J Transl Med 12: 4, 2014.

17. Das SK and Hoefler G: The role of triglyceride lipases in cancer associated cachexia. Trends Mol Med 19: 292-301, 2013.

18. Louie SM, Roberts LS, Mulvihill MM, Luo K and Nomura DK: Cancer cells incorporate and remodel exogenous palmitate into structural and oncogenic signaling lipids. Biochim Biophys Acta 1831: 1566-1572, 2013.

19. Alonso R, Mazzeo C, Mérida I and Izquierdo M: A new role of diacylglycerol kinase alpha on the secretion of lethal exosomes bearing Fas ligand during activation-induced cell death of $\mathrm{T}$ lymphocytes. Biochimie 89: 213-221, 2007.

20. Prinz PU, Mendler AN, Masouris I, Durner L, Oberneder R and Noessner E: High DGK- $\alpha$ and disabled MAPK pathways cause dysfunction of human tumor-infiltrating $\mathrm{CD}^{+} \mathrm{T}$ cells that is reversible by pharmacologic intervention. J Immunol 188: 5990-6000, 2012.

21. Dominguez CL, Floyd DH, Xiao A, Mullins GR, Kefas BA, Xin W, Yacur MN, Abounader R, Lee JK, Wilson GM, et al: Diacylglycerol kinase $\alpha$ is a critical signaling node and novel therapeutic target in glioblastoma and other cancers. Cancer Discov 3: 782-797, 2013.

22. Jia YF, Xiao DJ, Ma XL, Song YY, Hu R, Kong Y, Zheng Y, Han SY, Hong RL and Wang YS: Differentiated embryonic chondrocyte-expressed gene 1 is associated with hypoxia-inducible factor $1 \alpha$ and Ki67 in human gastric cancer. Diagn Pathol 8: 37, 2013.

23. Shi XH, Zheng Y, Sun Q, Cui J, Liu QH, Qü F and Wang YS: DEC1 nuclear expression: A marker of differentiation grade in hepatocellular carcinoma. World J Gastroenterol 17: 2037-2043, 2011.

24. Liu Y, Wang L, Lin XY, Wang J, Yu JH, Miao Y and Wang EH: The transcription factor DEC1 (BHLHE40/STRA13/SHARP-2) is negatively associated with TNM stage in non-small-cell lung cancer and inhibits the proliferation through cyclin D1 in A549 and BE1 cells. Tumour Biol 34: 1641-1650, 2013. 